El maltrato estructural a personas mayores en Chile y la necesidad de formular un índice multidimensional Carolina Riveros - Patricia Rodríguez - Rodrigo Palomo - Sandra Alvear - M. Ángeles Fernández - Ángela Arenas Pp. 163-176

\title{
EL MALTRATO ESTRUCTURAL A PERSONAS MAYORES EN CHILE Y LA NECESIDAD DE FORMULAR UN ÍNDICE MULTIDIMENSIONAL
}

\author{
The structural maltreatmen to eldery in Chile \\ and the need of formulate a multidimensional index
}

\author{
Carolina Riveros* \\ Patricia Rodríguez** \\ Rodrigo Palomo*** \\ Sandra Alvear**** \\ M. Ángeles Fernández***** \\ Ángela Arenas******
}
* Facultad de Ciencias Jurídicas y Sociales, Universidad de Talca, Santiago, Chile. Trabajo escrito en el marco del Proyecto FONDEF ID16AM0006, titulado: "El maltrato estructural a las personas mayores en Chile. Formulación de un índice multidimensional" de la cual la autora es la investigadora responsable. Correo electrónico:criveros@utalca.cl
** Facultad de Economía y Negocios, Universidad de Talca. Santiago, Chile. Correo electrónico: prodrig@utalca.cl
***Facultad de Ciencias Jurídicas y Sociales, Universidad de Talca. Talca, Chile. Correo electrónico: rpalomo@utalca.cl
**** Facultad de Economía y Negocios, Universidad de Talca. Talca, Chile. Correo electrónico: salvear@utalca.cl
***** Facultad de Ciencias Jurídicas y Sociales, Universidad de Talca. Santiago, Chile mangeles@ haymujeres.cl
****** Facultad de Ciencias Jurídicas y Sociales, Universidad de Talca. Santiago, Chile. Correo electrónico: aarenas@utalca.cl

Agradecemos muy especialmente a Gabriela Villarroel y Maximiliano Olivares por la revisión de los aspectos formales de este artículo.

Artículo recibido el 07 de marzo de 2017. Aceptado el 28 de agosto de 2017. 


\title{
RESUMEN
}

El trabajo pretende sentar las bases de problematización dogmática y aplicativa sobre el maltrato estructural hacia personas mayores, tema complejo, contingente, ampliamente extendido en nuestras sociedades y que suele invisibilizarse. Al efecto, se propone una descripción crítica de las principales manifestaciones socioculturales, jurídicas y económicas del maltrato estructural. En particular, se analiza el binomio entorno-adulto mayor, el panorama normativo nacional e internacional, y su traducción económica en el mercado de trabajo y el sistema de protección social. De otra parte, atendida la complejidad del fenómeno y las peculiaridades del caso chileno, se justifica la necesidad urgente de formular un índice multidimensional que aporte a la solución del problema de visibilización y caracterización del maltrato estructural.

Palabras clave: adulto mayor, maltrato estructural, índice multidimensional.

\begin{abstract}
The paper aims to establish the bases of dogmatic and applicative problematization about structural maltreatment towards elderly, complex topic, contingent, widely spread in our societies and often invisible. To this end, a critical description of the main sociocultural, legal and economic manifestations of structural maltreatment is proposed. In particular, the binomial environment-elderly is analyzed, the national and international normative panorama, and its economic repercussions into the labor market and the social protection system. On the other hand, given the complexity of the phenomenon and the peculiarities of the Chilean case, the urgent need to formulate a multidimensional index that contributes to the solution of the problem of visualization and characterization of structural maltreatment is argued.
\end{abstract}

Keywords: Elderly people, structural abuse, multidimensional index.

\section{INTRODUCCIÓN}

En este trabajo nos referiremos al maltrato estructural hacia las personas mayores. SENAMA ha definido este maltrato como: "Aquel que ocurre desde y en las estructuras de la sociedad mediante normas legales, sociales, culturales, económicas que actúan como trasfondo de todas las otras formas de maltrato existente" (SENAMA, 2005).

En consecuencia es una temática muy compleja en la que el factor de la invisibilidad desempeña un rol preponderante. Es un tipo de violencia silenciosa que no por ello resulta menos nociva (La Parra y Tortosa, 2003). Además, la problemática 
es actual, puesto que, es posible percatarse de cómo la sociedad mundial, pero particularmente la sociedad chilena envejece. En Chile la situación es preocupante, ya que, en el 2050 se proyecta que en nuestro país habrá 6,3 millones de personas con más de 60 años, lo que corresponde al 29,5 \% de la población chilena (Comisión Asesora Presidencial sobre el Sistema de Pensiones, en adelante, Comisión Bravo, 2015); Inglés (2016). A nivel mundial y latinoamericano el envejecimiento es similar (NACIONES UNIDAS, 2014; Olivi et al., 2015). Asimismo, respecto a este segmento etario se produce una tensión dialéctica entre su autonomía CASEN (2013) y la protección que debe otorgársele. Se requiere por ello, una adecuada simbiosis entre fuerzas antagónicas: por una parte la autonomía de la persona y por otro lado su protección.

El objetivo de este trabajo es abordar la necesidad de profundizar en el fenómeno del maltrato estructural hacia el adulto mayor desde lo conceptual y lo cuantitativo Osorio et al., (2011). Para lo anterior, se propone la medición de esta tipología de maltrato a través de un instrumento multidimensional que permita su caracterización. El trabajo se estructura de la siguiente manera: En primer lugar, se explicitarán algunas manifestaciones del maltrato estructural; enseguida se revisará el estado de la cuestión en instrumentos de medición aplicados a personas mayores y finalmente se concluye en la necesidad de la creación un índice multidimensional que mida el maltrato estructural hacia el adulto mayor.

\section{MANIFESTACIONES DEL MALTRATO ESTRUCTURAL AL ADULTO MAYOR}

El problema del maltrato estructural es cotidiano y abarca distintos ámbitos como por ejemplo: sociocultural, jurídico y económico. Por consiguiente, se violenta sistemáticamente a un segmento importante y cada vez más creciente de nuestra sociedad que se le desvaloriza a través de convencionalismos sociales o cuando se incumplen leyes existentes o cuando existe carencia o mala distribución de los recursos económicos encaminados a una integración plena de las personas mayores (Letelier, 2005; Trejo, 2001; Zapata, 2001).

\section{ÁMBITO SOCIOCULTURAL}

Se refiere al maltrato estructural que se desarrolla "en" la sociedad. En este sentido el concepto "entorno" alude al ambiente próximo que rodea al mayor. También al conjunto de características que define el espacio público donde la persona se desenvuelve y a la forma en que otros grupos etarios interactúan en este ambiente colectivo. Aparece el entorno como un nivel que surge de manera 
espontánea, donde la casa y la calle son dos espacios simbólicos que establecen fronteras que van desde lo privado a lo público.

De este modo, estudios seńalan que uno de los contextos consensuados para interpretar el maltrato es el orden familiar más allá de la casa, donde se concentran acontecimientos que se pueden calificar de maltrato (Arenas, 2012; SENAMA, 2014). La calle aparece como un espacio que no remite a lo doméstico o a los vínculos familiares y construye en cambio, otros lugares en los que se pueden experimentar violencias de diferentes características, algunas de las cuales no son exclusivamente dirigidas al mayor (delincuencia, transporte público, infraestructura, etc.).

Cabe destacar que a nivel comunitario o de entorno, algunas variables que pueden asociarse al maltrato son aquellas que surgen de los procesos de modernización, tales como la erosión de estructuras familiares tradicionales y sus dificultades para cumplir con las tareas de seguridad y protección.

Desde esta perspectiva, la consecuencia más grave del maltrato es el aislamiento de la persona mayor, la disminución de su autoestima y los sentimientos de inseguridad que a la larga favorecen la instalación de estereotipos negativos de la vejez asociados a la desvinculación y a la falta de proyectos individuales (Huenchuán, 2014; SENAMA, 2014; Thumala, 2016).

Estas tipologías de violencias son públicas y necesariamente se relacionan con el grado y calidad en las interacciones sociales en las que los adultos mayores participan.

\section{ÁMBITO JURÍDICO}

Desde esta perspectiva el problema esencial es que las normas jurídicas no se adecúan al proceso de envejecimiento poblacional ni individual. Por otro lado, la dispersión normativa influye sustancialmente en el actuar de los operadores jurídicos y confunde a la población, que en definitiva desconoce los derechos de la persona mayor. En este sentido, un problema relevante es el reconocimiento de la autonomía de la persona (Barcia, 2014; Bórquez et al., 2007; Lathrop, 2009); Riveros et al., 2015). En términos generales, la autonomía dice relación con la facultad de autodeterminación que poseen los individuos y con las posibilidades de ejercicio de sus derechos, que ha sido ampliamente estudiada en el Derecho Comparado (Alventoso, 2014; Dodegge, 2013; Duttge et al., 2015; Fernandez De Buján, 2011; Klein, 2005; Rams, 2011; Ruz, 2013; Schwab, 2013). La autonomía es una dimensión que concierne el diario vivir de los adultos mayores por cuanto se manifiesta a lo largo de sus rutinas diarias Osorio et al., (2011). Respecto a la temática de la capacidad las normas legales existentes en el Código Civil son anacrónicas. No existen disposiciones legales adecuadas que permitan acompañar 
a las personas en el proceso de envejecimiento. Las actuales normas presentan una dicotomía. Se es capaz o incapaz. Sin embargo, esta dualidad no se condice con el proceso de envejecimiento. En algunas personas mayores pueden generarse dolencias psíquicas o físicas y, la ley solo contempla una causal de incapacidad fundada en la demencia. Ello no es lo suficientemente dúctil para la realidad de los adultos mayores en la actualidad.

La complejidad de la vejez también incorpora perspectivas jurídicas y bioéticas (Barrantes-Monge et al., 2009; Arenas, 2012); respecto de la doctrina comparada, se pueden considerar entre otros: Damm, 2015; Pérez-Jara, 2011; Perlingeiro, 2014; Spickhoff, 2008; Taupitz, 2008, 2012. Específicamente los cuerpos legales relativos a estos temas a saber, Ley 20.584; Ley 20.120, entre otros, también contienen ejemplos de maltrato estructural a las personas mayores. En este ámbito no se entrega herramientas adecuadas para acompañar a las personas en su proceso de envejecimiento. El artículo 28, inciso $1^{\circ}$ de la Ley $\mathrm{N}^{\circ} 20.548$, impide que una persona con discapacidad psíquica o intelectual, que no pueda expresar su voluntad, pueda participar en una investigación científica, vale decir, las personas mayores (que se encuentren en esta situación) solo pueden participar en investigaciones científicas si en tiempo real prestan su consentimiento. Ello es una manifestación del régimen de incapacidad del Código Civil de 1857 y de su asincronía con normas internacionales vigentes. De esta manera esta disposición atenta contra el artículo 25 de la Convención de Naciones Unidas sobre Derechos de las Personas con Discapacidad, que exige a los Estados prevenir y reducir al máximo la aparición de nuevas discapacidades, incluidas las que se presentan en personas mayores en situación de discapacidad mental (Riveros et al., 2015).

\section{ÁMBITO ECONÓMICO}

Desde la perspectiva económica y financiera, contar con una población longeva, tiene una doble mirada desde los indicadores de salud pública, es una muy buena señal, sin embargo, desde la economía plantea importantes desafíos. Existen estudios a nivel internacional que muestran dos importantes desventajas asociadas al envejecimiento. La primera es financiera: los costos de salud y jubilación suben cuando la población de más de 65 años aumenta más rápido que la población activa (Calvo et al., 2008). Lo descrito, implica que las personas deben utilizar gran parte de sus ingresos en medicamentos y prestaciones sanitarias. También, considera la disminución abrupta de ingresos una vez alcanzada la jubilación o incluso la falta de ella, lo que conlleva el acceso a pensiones básicas solidarias. La segunda desventaja se relaciona con la equidad entre generaciones, es decir, cuanto más alto es el porcentaje de ancianos, más probable es que los trabajadores actuales y sus descendientes paguen 
una mayor proporción de sus ingresos a los actuales ancianos, y menos probable que reciban prestaciones similares en su vejez. Se plantea que la única forma de reducir la carga para las generaciones futuras es pagar más impuestos hoy y, recibir menos prestaciones relacionadas con la seguridad social. Así, se reduciría el aumento previsto del déficit y se distribuiría el ajuste equitativamente con las generaciones futuras (Batini et al., 2011). En Chile, debido a la baja tasa de natalidad y al aumento de las expectativas de vida, hacia fines de los años 70 se proyectó un desequilibrio entre trabajadores activos y pensionados lo que constituyó una de las principales causas para cambiar el sistema en el año 1981, basado en una contribución mínima obligatoria del 10\% mensual (Balbontín, 2016). En tal sentido, la Comisión Bravo (2015), frente a una nueva reforma, entre sus principales recomendaciones propone: incrementar el monto de las pensiones autofinanciadas, mejorar las pensiones contributivas, aumentar la edad legal de jubilación e incentivar el trabajo de las personas mayores (Aparicio et al., 2007; Cabeza et al., 2009).

En torno a las políticas públicas de Chile respecto de las personas mayores se puede observar que se ha buscado transformar la visión asistencialista de antaño para dar paso a una visión que permita el ejercicio de derechos a este segmento etario; con todo, existen una serie de temas todavía pendientes. Se puede observar las siguientes políticas públicas vigentes:

TABLA Nº 1 . Políticas públicas dirigidas a los adultos mayores

\begin{tabular}{|l|l|c|}
\hline \multicolumn{1}{|c|}{ Ministerio } & \multicolumn{1}{|c|}{ Política } & Inicio \\
\hline $\begin{array}{l}\text { Ministerio de Desarrollo Social. } \\
\text { Servicio Nacional del Adulto Mayor }\end{array}$ & Programa de asesores seniors & 2003 \\
\hline $\begin{array}{l}\text { Ministerio de Desarrollo Social. } \\
\text { Servicio Nacional del Adulto Mayor }\end{array}$ & Programa Participación y Formación & 2007 \\
\hline $\begin{array}{l}\text { Ministerio de Desarrollo Social } \\
\text { Servicio Nacional del Adulto Mayor }\end{array}$ & Programa de envejecimiento activo & 2013 \\
\hline $\begin{array}{l}\text { Ministerio de Desarrollo Social. } \\
\text { Servicio Nacional del Adulto Mayor }\end{array}$ & $\begin{array}{l}\text { Fondo nacional del adulto mayor para } \\
\text { proyectos autogestionados }\end{array}$ & 2002 \\
\hline $\begin{array}{l}\text { Ministerio de Desarrollo Social. } \\
\text { Servicio Nacional del Adulto Mayor }\end{array}$ & $\begin{array}{l}\text { Fondo de Servicios de Atención al Adulto } \\
\text { Mayor: Condominios de Viviendas Tuteladas }\end{array}$ & 2011 \\
\hline $\begin{array}{l}\text { Ministerio de Desarrollo Social. } \\
\text { Servicio Nacional del Adulto Mayor }\end{array}$ & Programa de turismo social & 2009 \\
\hline $\begin{array}{l}\text { Ministerio de Desarrollo Social. } \\
\text { Servicio Nacional del Adulto Mayor }\end{array}$ & Sistema Nacional de apoyo y cuidados & 2016 \\
\hline $\begin{array}{l}\text { Ministerio de Desarrollo Social. } \\
\text { Servicio Nacional del Adulto Mayor }\end{array}$ & Fondo para ejecutores intermedios. & 2016 \\
\hline $\begin{array}{l}\text { Ministerio de Desarrollo Social. } \\
\text { Servicio Nacional del Adulto Mayor }\end{array}$ & $\begin{array}{l}\text { Fondo de Servicios de Atención al Adulto } \\
\text { Mayor: Establecimientos de Larga Estadia }\end{array}$ & 2013 \\
\hline
\end{tabular}




\begin{tabular}{|c|c|c|}
\hline $\begin{array}{l}\text { Ministerio de Desarrollo Social. } \\
\text { Servicio Nacional del Adulto Mayor }\end{array}$ & Programa de Cuidados Domiciliarios & 2013 \\
\hline $\begin{array}{l}\text { Ministerio de Desarrollo Social. } \\
\text { Servicio Nacional del Adulto Mayor }\end{array}$ & Programa Centros de Día & 2012 \\
\hline $\begin{array}{l}\text { Ministerio de Desarrollo Social. } \\
\text { Servicio Nacional del Adulto Mayor }\end{array}$ & $\begin{array}{l}\text { Programa Subvención a ELEAM sin fines } \\
\text { de lucro }\end{array}$ & 2013 \\
\hline $\begin{array}{l}\text { Ministerio de Desarrollo Social. } \\
\text { Servicio Nacional del Adulto Mayor }\end{array}$ & $\begin{array}{l}\text { Programa Escuela para funcionarios } \\
\text { públicos }\end{array}$ & 2009 \\
\hline $\begin{array}{l}\text { Ministerio de Desarrollo Social. } \\
\text { Servicio Nacional del Adulto Mayor }\end{array}$ & Programa buen trato al adulto mayor & 2012 \\
\hline $\begin{array}{l}\text { Ministerio de Desarrollo Social. } \\
\text { Servicio Nacional del Adulto Mayor }\end{array}$ & Programa Vinculos & 2012 \\
\hline Ministerio de Economía & Programa de vacaciones tercera edad & 2001 \\
\hline Ministerio de Salud & $\begin{array}{l}\text { Examen de Medicina Preventiva } \\
\text { (EMPAM) }\end{array}$ & 2005 \\
\hline Ministerio de Salud & Garantías Explícitas en Salud (GES) & 2006 \\
\hline Ministerio de Salud & $\begin{array}{l}\text { Programa nacional de alimentación } \\
\text { complementaria (PACAM) }\end{array}$ & 1999 \\
\hline Ministerio de Salud & Programa Nacional de Inmunizaciones & 1978 \\
\hline Ministerio de Salud & Programa de Camas Sociosanitarias & 2015 \\
\hline Ministerio de Salud & Programa de atención a la dependencia & 2006 \\
\hline Ministerio de Salud & Plan Nacional de demencias para Chile & 2017 \\
\hline Ministerio de Salud. & Programa Más adultos mayores autovalentes & 2015 \\
\hline $\begin{array}{l}\text { Ministerio del trabajo y previsión } \\
\text { social }\end{array}$ & Bono invierno & 2009 \\
\hline $\begin{array}{l}\text { Ministerio del trabajo y previsión } \\
\text { social }\end{array}$ & Bodas de oro & 2011 \\
\hline $\begin{array}{l}\text { Ministerio del trabajo y previsión } \\
\text { social }\end{array}$ & $\begin{array}{l}\text { Eliminación del 5\% de la cotización en } \\
\text { salud }\end{array}$ & 2016 \\
\hline $\begin{array}{l}\text { Ministerio del trabajo y previsión } \\
\text { social }\end{array}$ & $\begin{array}{l}\text { Fortalecimiento Oficinas Municipales de } \\
\text { Información Laboral (FOMIL) }\end{array}$ & 2009 \\
\hline $\begin{array}{l}\text { Ministerio del trabajo y previsión } \\
\text { social }\end{array}$ & $\begin{array}{l}\text { Servicio Nacional de Capacitación y } \\
\text { Empleo. Sence }\end{array}$ & 1976 \\
\hline Ministerio del Deporte & Adulto mayor en movimiento & 2012 \\
\hline Ministerio de Hacienda & $\begin{array}{l}\text { Rebaja del impuesto territorial para } \\
\text { personas mayores }\end{array}$ & 2014 \\
\hline $\begin{array}{l}\text { Ministerio de Vivienda y } \\
\text { Urbanismo }\end{array}$ & Ley No20.422 & 2010 \\
\hline $\begin{array}{l}\text { Ministerio de Vivienda y } \\
\text { Urbanismo }\end{array}$ & Decreto Supremo No49 del 2012 & 2012 \\
\hline
\end{tabular}

Fuente: Elaboración propia. 


\section{LA NECESIDAD DE LA FORMULACIÓN DE UN ÍNDICE MULTIDIMENSIONAL DE MALTRATO ESTRUCTURAL EN CHILE}

Un índice multidimensional del maltrato de los adultos mayores debiese aportar a resolver el problema de visibilización y caracterización del maltrato estructural EURICH et al. (2015). Existen algunos estudios multidimensionales que caracterizan determinados y específicos aspectos relativos a la realidad de los adultos mayores en Chile a nivel internacional, nacional y local. Ninguno de ellos mide el maltrato estructural de los adultos mayores en Chile. A modo ejemplar: (i) Nivel internacional: a) The Global AgeWatch Index" (HelpAge International, 2013) constituye un índice global de envejecimiento; The Global AgeWatch Index evalúa los factores que determinan el bienestar social y económico de las personas adultas mayores en todo el mundo. Además de un análisis global, se ha enfocado en las diferentes regiones, atendiendo a las necesidades de las personas mayores y considerando la gran variedad de tendencias geográficas. Sus factores de medición son la seguridad de ingresos, el estado de salud, las competencias y el entorno favorable. b) El Índice de Envejecimiento, expresa la relación entre la cantidad de personas adultas mayores y la cantidad de niños y jóvenes. Considera la desagregación urbano-rural; según su interpretación convencional, se trata de un indicador asociado a las transferencias intergeneracionales y su aumento sistemático implica para los estados una mayor inversión en salud y seguridad social orientada a las personas de edad, beneficios de los cuales no deberían estar exentos los pueblos indígenas (CEPAL, 2012). c) El Índice de sospecha al maltrato de las personas mayores de Pérez-Rojo et al., (2009), busca desarrollar y validar un instrumento fiable para distintos contextos geográficos y culturales para aumentar la concienciación entre los profesionales de Atención Primaria sobre el maltrato y la negligencia hacia los ancianos, en caso de violencia física, psíquica, sexual, patrimonial o abandono (no incluye maltrato estructural). (ii) Nivel nacional: IDHAM, Índice de Desarrollo Humano para Adultos Mayores (Arenas, 2012); Cuarta Encuesta calidad de vida en la vejez (UC-Caja Los Andes, 2017), que contempla el Índice de Calidad de Vida en la Vejez y abarca las dimensiones de bienestar general en la vejez, situación económica y laboral, condiciones de salud, participación y actividades sociales, relaciones sociales y familiares y los cambios en el bienestar y los predictores de un buen envejecer. (iii) Nivel local. Calidad de vida especialmente lo relativo al entorno ambiental en Valparaíso (2015) que proviene del Fondecyt 1061179 y SENAMA Maltrato hacia las personas mayores en la Región Metropolitana (2013b). Esta investigación se centra en analizar el maltrato estructural a los adultos mayores en la sociedad chilena actual. De acuerdo a los datos de Conicyt en Chile el Programa de Fondef solo ha tenido como proyecto "Confección instrumento que detecte adultos mayores (a.m.) en riesgo 
de compromiso de funcionalidad - $\sin \mathrm{n}^{\circ}$ de proyecto" 1 . Y el programa Fondecyt a su vez posee el proyecto: "Calidad de vida del Adulto Mayor en el hábitat urbano: Estudio del caso de Valparaíso N ${ }^{\circ}$ de proyecto: 1061179”2.

De acuerdo a lo expuesto, no existe en Chile un mecanismo que mida el maltrato estructural. Por esta razón es relevante formular un índice multidimensional de maltrato estructural a los adultos mayores.

\section{CONCLUSIONES}

1. El maltrato hacia las personas mayores es un problema de relevancia social, sin embargo, está invisibilizado. Dicho problema es multicausal y puede verse manifestado en una serie de ámbitos.

2. Se reconocen manifestaciones de este maltrato en el ámbito sociocultural, en el ámbito jurídico y en el ámbito económico. En el primer ámbito, lo esencial está en el binomio entorno-persona mayor. Las consecuencias más peligrosas en este ámbito son las posibilidades de aislamiento, la disminución de autoestima y las emociones de inseguridad que favorecen la generación de estereotipos negativos de la vejez.

3. En el ámbito jurídico un problema fundamental es la falta de normativa acorde al proceso de envejecimiento poblacional y de carácter individual. Esencial resulta que se reconozca la autonomía de las personas que conforman este grupo etario. Respecto a la temática de la capacidad las normas legales existentes en el Código Civil son anacrónicas y asincrónicas a la normativa internacional. No existen suficientes disposiciones legales que permitan acompañar a las personas en el proceso de envejecimiento individual.

4. En el ámbito económico cuando la población de adultos mayores aumenta los costos de salud y jubilación también acrecen. Los ingresos no alcanzan y se utilizan en gran medida para financiar medicamentos y prestaciones de carácter sanitarias. Fundamental es en este plano, revisar el aumento de la edad legal de jubilación e incentivar el mercado de trabajo de las personas mayores.

\footnotetext{
${ }^{1}$ http://dspace2.conicyt.cl/handle/10533/17081

${ }^{2}$ http://dspace2.conicyt.cl/handle/10533/16256
} 
5. Se han descrito someramente algunos estudios multidimensionales que caracterizan determinados y específicos aspectos relativos a la realidad de los adultos mayores en Chile a nivel internacional, nacional y local. Sin embargo, ninguno de ellos mide de forma específica el maltrato estructural. Por consiguiente, es importante considerar que todas estas manifestaciones del maltrato estructural no han sido analizadas y medidas por un instrumento único multidimensional que específicamente analice cómo la sociedad, las normas legales y económicas invisibilizan y por ende, maltratan de forma estructural a las personas mayores.

6. Al revisar las políticas públicas en conjunto con los ámbitos de maltrato estructural queda en evidencia la necesidad de seguir progresando respecto del bienestar de los adultos mayores en Chile. Es por ello, que resulta adecuado generar un instrumento específico que mida cómo se está actualmente violentando de forma sistémica a las personas mayores en Chile y con ello contribuir a la generación de nuevas políticas públicas para este grupo etario.

\section{REFERENCIAS}

Alventoso, Josefina. "La incapacitación en España”, Revista Boliviana de Derecho $\mathrm{N}^{\circ} 17$ (2014): 252-275.

Aparicio, Joaquín y Olmo, Ana Marta. La edad como factor de tratamientos desiguales en el trabajo. España: Bomarzo, 2007.

Arenas, Ángela. Adulto Mayor: nuevas perspectivas para el desarrollo humano. Santiago de Chile: Ediciones Universidad Finis Terrae, 2012.

Arenas, Jessica. "El adulto mayor víctima de violencia intrafamilar. Su abordaje en los tribunales de familia” (SENAMA, 2014). Disponible en: http:// www.senama.cl/filesapp/Libro\%20Maltrato\%20Haciendo\%20visible\%20 FINAL\%20WEB\%2014\%20DE\%20MARZO.pdf

Balbontín, Renato. "Restricción de retornos mínimos, su impacto en los fondos de pensiones en Chile", Revista Internacional Administración \& Finanzas, vol.9 (2016): 1-13. 
Barcia, Rodrigo. "Algunas críticas al Derecho Común y especialmente a la regulación de las incapacidades respecto del adulto mayor en el ordenamiento jurídico chileno", Revista Chilena de Derecho Privado N²3 (2014): 57-86.

Barrantes-Monge, Melba, Rodríguez, Eduardo y Lama, Alexis. "Relación médico-paciente: Derechos del adulto mayor", Acta bioethica [en línea], vol. 15, No 2 (2009): 216-221. Disponible en: http://www.scielo.cl/scielo. php?script=sci_arttext\&pid=S1726569X2009000200013\&lng=es\&nrm= iso. [Consultado el 20 de mayo de 2016].

Batini, Nicoletta y Callegari, Giovanni. "Enfrentar con sabiduría los costos de envejecer", Finanzas \& Desarrollo. Publicación trimestral del Fondo Monetario Internacional y del Banco Mundial, 48/2 (2011): 19-21.

Bórquez, Gladys, Raineri, Gina, Horwitz, Nina y Huepe, Gabriela. "La noción de capacidad de la persona para tomar decisiones, en la práctica médica y legal”, Revista Médica de Chile N¹35 (2007): 1153-1159.

Cabeza, Jaime, Ballester, María Amparo y Fernández, Marta (Dir.). La relevancia de la edad en la relación laboral y de Seguridad Social. España: Thomson Reuters, 2009.

Calvo, Esteban y Martorell, Bernardo. "La salud del adulto mayor en Chile: una responsabilidad compartida por las personas, las empresas y el Estado", Foco N 130 (2008): 1-16. [en línea]. Disponible en: http://www.expansiva. cl/publicaciones/en_foco/detalle.tpl?iddocumento $=08072008171655$. [Consultado el 04 de junio de 2016].

\section{CASEN 2013}

Comisión Asesora Presidencial sobre el Sistema de Pensiones [versión web] [Consultado 25 de abril de 2016]. Disponible en: http://www.comisionpensiones.cl/Documentos/GetInforme. Damm, Reinhard. "Einwilligungsund Entscheidungsfaehigkeit in der Entwicklung von Medizin und Medizinsrecht", MedR No 33 (2015): 775-785.

Dodegge Aerztliche Zwangsmassnahmen und Betreuungsrecht en NJW. [en línea] 2013. Disponible en: http://beck-online.beck.de/Bcid/Y-300-Z-NJW-B2013-S-1265-N-1. 
Duttge y Simon. "Zwangsbehandlung kraft Betreuungsrechts?” en ZPR. [en línea] 2015. Disponible en: https://beck-online.beck.de/Bcid/Y-300-Z-ZRP-B2015-S-176-N-1.

Eurich, J., Nuessel, F., Wahl, H-W. y Oswald, F. "Struktural Lag" und Moeglichkeitsraeume des Alters en Zeitschrift fuer Gerontologie und Geriatrie, 48 (2015): 675-676.

Fernández De Buján, Antonio. "Capacidad. Discapacidad, Incapacitación. Modificación judicial de la capacidad”, RJUAM Nº 23 (2011): 53-81.

Huenchuán, Sandra. "El maltrato de las personas mayores: conceptos, normas y experiencias de políticas en el ámbito internacional (SENAMA, 2014). Disponible en: http:/www.senama.cl/filesapp/Libro\%20Maltrato\%20 Haciendo\%20visible\%20FINAL\%20WEB\%2014\%20DE\%20MARZO. pdf.

Human Development Report (HDR) Human Development Report 2013: The Rise of the South Human progress in a Diverse World - Technical Notes, UNDP, New York, 2013.

Inglés, Rayén. "Políticas públicas sobre envejecimiento: demografía, salud e impacto social”. En Palomo et al. (Eds). Talca, (2016): 27-41.

Klein, H. (2005) “Fester Aberglaube statt sicheren Wissens” RDG [en línea] 2005. Disponible en: https://beck-online.beck.de/Bcid/Y-300-Z-RDG-B-2005S-78-N-1. [Consultado el 20 de mayo de 2016].

La Parra, Daniel y Tortosa, José. "Violencia estructural: una ilustración del concepto", Documentación Social N¹31 (2003): 57-72.

Lathrop, Fabiola. "Protección jurídica de los adultos mayores en Chile", Revista Chilena de Derecho, 36/1 (2009): 77-113.

Letelier, Azucena. "Maltrato en la Vejez", Revista de Psicología de la Universidad de Chile, XIV/1 (2005): 99-112.

Naciones Unidas, Departamento de asuntos económicos y sociales. La situación demográfica en el mundo, 2014: Informe conciso. Nueva York, 2014. 
Observatorio Demográfico División de Población de la CEPAL, 2012.

Olivi, Alessandra, Fadda-Cori, Giulietta y Pizzi-Kirschbaum, Marcela. "Evaluación de la calidad de vida de los adultos mayores en la ciudad de Valparaíso", Papeles de Población No 84 (2015): 227-249.

Osorio, Paulina, Torrejón, María y Anigstein, María Sol. "Calidad de vida en personas mayores en Chile", Revista Mad de la Universidad de Chile $\mathrm{N}^{\circ} 24$ (2011): 61-75.

Pérez-Jara, Javier. "Cuestiones éticas en los pacientes mayores hospitalizados. Experiencia en el Reino Unido”, Cuadernos de Bioética XXII (2011): 517-533.

Pérez-Rojo, Gemma, Izal, María, Montorio, Ignacio, Regalto, Pilar, Espinoza, Juan Manuel. "Prevalencia de malos tratos hacia personas mayores que viven en la comunidad en Espańa”, Med Clin (Barc). 2013. Disponible en: http:// dx.doi.org/10.1016/j.medcli.2012.09.048, (2009): 1-5.

Perlingeiro, Ricardo. "Los Cuidados de salud para los ancianos entre las limitaciones presupuestarias y el derecho a un mínimo existencial”, Boletín Mexicano de Derecho Comparado ańo XLVII N¹40 (2014): 547-584.

Rams, Joaquín. "Hombre y persona. Personalidad. Capacidad e incapacidad. Discapacidad y vejez", Revista Critica de Derecho Inmobiliario N 723 (2011): 211-296.

Riveros, Carolina y Barcia, Rodrigo, "La Obligación ética y jurídica de prevenir y reducir al máximo la aparición de nuevas discapacidades en las personas mayores en Chile", Acta Bioethica, 21/2 (2015): 207-215.

Ruz Lártiga, Gonzalo. "El mandato de protección futura. Mirada a una medida propuesta por el derecho civil francés para personas en situación de ser protegidas”. En Henriquez, Ian (coord.). El mandato. Ensayos doctrinarios y comentarios de jurisprudencia. Santiago: Thomson Reuters Abeledo Perrot, (2013):5-30.

Schwab, Dieter. Familienrecht 21. Auflage: Verlag C.H. Beck, 2013.

SENAMA 2005 [versión web]. Disponible en: http://www.senama.cl/filesapp/1288. pdf. [Consultado el 20 de mayo de 2016] 
SENAMA 2014 [Versión web]. Disponible en: http://www.senama.cl/filesapp/ Libro\%20Maltrato\%20Haciendo\%20visible\%20FINAL\%20WEB\%20 14\%20DE\%20MARZO.pdf. [Consultado el 20 de mayo de 2016].

SENAMA 2015 [Versión web]. Disponible en: http://www.senama.cl/filesapp/ interiorSenamaOKFINAL.pdf. [Consultado el 20 de mayo de 2016].

Spickhoff, Andreas. "Selbstbestimmung im Alter - Möglichkeiten und Grenzen", ZfRV N 5 (2008): 33-41.

Taupitz, Jochen. "Selbstbestimmungsrecht des Patienten: Aktuelle Kontroversen im deutschen Recht”. En Dogan H, Mahmutoglu F, Namal A, (Eds.). Türkisches Jahrbuch für Studien zu Ethik und Recht in der Medizin (2008): 43-52.

Taupitz, Jochen. "Medizinische Forschung an jungen und alten Patienten", MedR N³0 (2012): 583-588.

Thumala, Daniela. "Impacto de la exclusión social en la salud de los adultos mayores". En Palomo, Iván y Giacaman, Rodrigo (Eds.). Envejecimiento: demografía, salud e impacto social. Talca: Universidad de Talca (2016): 195202.

Trejo, Carlos. "Elviejo en la historia”, Actabioethica, 7/1 (2001):107-119.Disponible en: http://www.scielo.cl/scielo.php?script=sci_arttext\&pid=S1726-569X2 001000100008\&lng=es\&nrm=iso. [Consultado 20 de mayo de 2016].

Zapata, Hernán. "Adulto Mayor: Participación e Identidad”, Revista de Psicología de la Universidad de Chile, X/1(2001):189-197. 